\title{
Solidaridad y geopolítica. La Unión Europea como actor global de desarrollo
}

\section{Iliana Olivié', María Santillán²}

Resumen: Como donante de ayuda al desarrollo, la Unión Europea es la suma de las instituciones europeas y cada uno de los Estados miembro. Como tal, los objetivos, motivos y prioridades de su política de cooperación al desarrollo son diversos, y evolucionan con la realidad cambiante que rodea a la Unión. Acorde con la agenda global de desarrollo, la ayuda de la UE se ha alineado con los principios del desarrollo humano y, posteriormente, sostenible. Sin embargo, estos paradigmas han interactuado también con una creciente presencia de objetivos geopolíticos en la ayuda, que se han reflejado además en sus prioridades geográficas (con un mayor foco en la vecindad europea) y sectoriales. Paralelamente, se ha reforzado el énfasis en los principios de eficacia y coherencia, a lo que se ha tratado de dar respuesta con medidas que profundicen la integración europea. Todas estas tendencias se han visto reflejadas en la evolución de los instrumentos europeos para la cooperación, varios de los cuales se han fusionado recientemente en el Instrumento de Vecindad, Desarrollo y Cooperación Internacional.

Palabras clave: UE, cooperación internacional, ayuda, desarrollo, NDICl.

Solidarity and geopolitics. The European Union as a global development actor

Abstract: As a global aid sponsor, the European Union is the sum of all the European institutions and all the member states. As
Solidarité et géopolitique. L'Union Européenne comme acteur mondiale de développement

Résumé: En tantque bailleur defonds mondial, I'Union européenne est la somme de toutes les

\footnotetext{
${ }^{1}$ Investigadora principal, Real Instituto Elcano.

${ }^{2}$ Investigadora, Real Instituto Elcano.
} 
such, the objectives, motives and priorities of its policies regarding development cooperation are diverse and evolve depending on the changing realities surrounding the EU. According to the global developmentagenda, the aid provided by the EU has aligned itself in accordance the principles of human, and later, sustainable development. However, these paradigms have been interacting also with a growing presence of geopolitical objectives behind the aid, which has been reflected in its geographical and sectoral priorities (with a greater focus on the European neighbourhood). At the same time, principles of effectiveness and coherence have been strengthened by introducing measures that strengthen European integration. All these tendencies have been reflected in the evolution of European instruments for cooperation, several of which have channelled together to create the $\mathrm{NDICl}$.

Key words: EU, international cooperation, aide, development, NDICI. institutions européennes et de tous les États membres. En tantquetels, les objectifs, les motifs et les priorités de ses politiques en matière de coopération au développement sont divers et évoluent en fonction des réalités changeantes de I'UE. Selon l'agenda mondial du développement, I'aide fournie par l'UE s'est alignée sur les principes du développement humain et, plus tard, du développement durable. Toutefois, ces paradigmes ont également interagi avec la présence croissante d'objectifs géopolitiques derrière l'aide, qui s'est reflétée dans ses priorités géographiques et sectorielles (avec un accent plus marqué sur le voisinage européen). En même temps, les principes d'efficacité et de cohérence ont été renforcés parl'introduction de mesures renforçantl' intégration européenne. Toutes ces tendances se sont reflétées dans l'évolution des instruments européens de coopération, dont plusieurs se sont rassemblés pour créer le NDICl.

Mots clé: UE, coopération internationale, aide, développement, NDICI.

\section{Introducción}

La Unión Europea (UE), como donante, es un extraño objeto político. A diferencia de otras políticas públicas que, en el marco europeo están completamente comunitarizadas (como la comercial) o no lo están en absoluto (como la de salud, al menos hasta el momento de la pandemia), la de cooperación al desarrollo estaría a mitad de camino, o semi-comunitarizada. Así pues, co-existen programas bilaterales y multilaterales, como las aportaciones a Naciones Unidas o al Banco Mundial, de los Estados miembro (EM) con los fondos que éstos canalizan a la UE y que ésta invierte en el Sur global vía sus propias herramientas e instituciones (Comisión Europea, Banco Europeo de Inversiones -BEI) y, por lo tanto, a través de sus propios objetivos y programas.

Como resultado de lo anterior, la ayuda europea se ha comportado en ocasiones como la suma de los 28 (ahora 27) fragmentos, a los que se sumarían las instituciones 
europeas (casi como un fragmento más) (Arts y Dickinson, 2004; Grimm, 2006; Lundsgaarde, 2011; OCDE, 2020). Esta problemática explicaría las iniciativas de integración (en su mayoría bottom-up) como Team Europe, la programación conjunta, o la ayuda delegada.

La ayuda (y, por lo tanto, su integración política) es una pieza esencial del rol global de la UE. Siendo el primer donante mundial por volumen de ayuda desembolsada, es, de hecho, uno de los pocos ámbitos en los que podría llegar a ejercer un fuerte liderazgo y contrapeso frente a los otros dos grandes actores globales, Estados Unidos y China (a diferencia de lo que ocurre, por ejemplo, en los campos de lo tecnológico o lo militar). Y, quizás por ello, a medida que se consolida la rivalidad estratégica entre Estados Unidos y China, la retórica y el enfoque de la ayuda de la UE han ido sumando crecientemente objetivos geopolíticos, incluso securitarios, al enfoque tradicional de la solidaridad, la erradicación de la pobreza y el desarrollo humano.

Este artículo analiza la cooperación al desarrollo de la UE desde la perspectiva de sus motivos y objetivos (primer epígrafe), su aparato institucional, y la distribución de la ayuda europea (segundo epígrafe); todo ello desde la doble perspectiva de su creciente integración y perfil político y del doble enfoque solidario y geopolítico.

\section{Objetivos de la cooperación de la Unión Europea}

Dada la particular naturaleza de la UE como donante, los objetivos estratégicos de la cooperación para el desarrollo europea podrían definirse de muy distintos modos. Contaríamos entre ellos los objetivos de las instituciones europeas pero también los de cada uno de sus EM. Una forma de poder capturar esa confluencia o conjunto de objetivos y motivos de la ayuda es con el análisis de los denominados consensos de desarrollo.

\section{I. El qué: objetivos y motivos de la ayuda}

En 2006, la UE publicó el Consenso Europeo para el Desarrollo (Europa, Consejo, Europa, Parlamento y Europa, Comisión, 2006), un texto en el que participaron las instituciones europeas, pero también cada uno de los EM, precisamente en un intento por incrementar la coordinación y armonización de la cooperación de la UE. Reflejaría, por tanto, de forma aproximada, el enfoque europeo del desarrollo. 
En él se recogen ocho objetivos: (1) erradicar la pobreza extrema y el hambre; (2) lograr la enseñanza primaria universal; (3) promover la igualdad de género y la autonomía de la mujer; (4) reducir la mortalidad infantil; (5) mejorar la salud materna; (6) luchar contra el $\mathrm{VIH} /$ sida, el paludismo y otras enfermedades; (7) garantizar la sostenibilidad y el medio ambiente; y (8) fomentar una asociación mundial para el desarrollo. Esto es, hace suyos los Objetivos de Desarrollo del Milenio (ODM) ${ }^{3}$ vigentes entre 2000-2015, con lo que la agenda del desarrollo europea coincidiría con la global, establecida desde el marco de Naciones Unidas.

Sin embargo, el Consenso de 2006 también hace referencia a elementos políticos y económicos que se esquivaron deliberadamente en la agenda de los ODM pero que reaparecerían algo más tarde en la agenda global de desarrollo sintetizada en la Agenda 2030: fomento de la buena gobernanza y respeto de los derechos humanos como valores comunes y cimientos de la UE o crecimiento económico y creación de riqueza en favor de las poblaciones más pobres. Asimismo, este documento estratégico hace referencia a principios democráticos como la asociación, el diálogo político o la participación de la sociedad civil.

Poco tiempo después de la publicación del Consenso de 2006 se sucederían una serie de acontecimientos históricos que cambiarían fuertemente la visión estratégica de la ayuda europea. Por supuesto, el Tratado de Lisboa (Unión Europea, 2007), que dotaría al proyecto europeo de un marco constitucional, pero también la Gran Recesión de 2008-2011, el auge de movimientos populistas y nacionalistas en varios EM o la mal llamada crisis de refugiados de mediados de los 2010. Todos estos hechos vendrían a moldear un discurso europeo que debía adaptarse, además, a una nueva agenda de desarrollo global, la Agenda 2030, más compleja y amplia (con 17 objetivos en lugar de 8) y también más multidisciplinar (sumando a la dimensión social de los ODM, la económica, la política y la medioambiental y climática) (Sachs, 2012; Le Blanc, 2015). ${ }^{4}$

Así, el Nuevo Consenso Europeo de Desarrollo, publicado en 2017 (Comisión Europea, 2017), define una ayuda europea centrada en (1) las personas (pobreza, desigualdad, desarrollo humano, igualdad de género, migración, cultura, educación o salud), (2) el planeta (medioambiente, transición ecológica, recursos

\footnotetext{
${ }^{3}$ Véanse los objetivos, las metas y su progreso en https://wwwl.undp.org/content/undp/es/home/ sdgoverview/mdg_goals.html

${ }_{4}^{4}$ Puede encontrarse el detalle de los Objetivos de Desarrollo Sostenible en: https://www.un.org/ sustainabledevelopment/es/objetivos-de-desarrollo-sostenible/
} 
naturales o energía sostenible), (3) la prosperidad (comercio, crecimiento sostenible o transformación digital), (4) la paz (democracia, Estado de Derecho, Derechos Humanos), y (5) la asociación (con miras a crear las alianzas necesarias con la sociedad civil o la comunidad de donantes bilaterales y multilaterales en aras del desarrollo global).

Por tanto, de nuevo, los objetivos estratégicos de la UE como donante son los suscritos por el conjunto de la comunidad internacional en el marco de las Naciones Unidas pero aquí también, de nuevo, un análisis pormenorizado de los objetivos y motivos declarados en el nuevo consenso revela otros elementos, algunos de ellos geopolíticos o geoestratégicos, asociados a la política de ayuda.

De hecho, un análisis de contenidos de ambos documentos revela que estos dos paradigmas (el del desarrollo social, encapsulado en los ODM, el del desarrollo sostenible, sintetizado en la Agenda 2030) coexisten con un tercer paradigma, el securitario. Esto es así, sin embargo, sin cambios reseñables entre los dos documentos: en proporciones casi invariables de en torno al $15 \%$ en ambos documentos, los objetivos en materia de cooperación incluyen menciones a los criterios de seguridad (notablemente, el control migratorio y la prevención de conflictos). Es más, estas son muy inferiores al peso de las alusiones a los paradigmas del desarrollo social (protagonista en el primer consenso) y del desarrollo sostenible (presente en cerca del $58 \%$ de las referencias a los objetivos de la ayuda en el consenso más reciente) (Olivié y Pérez, 2021). ${ }^{5}$

Estos dos documentos estratégicos recogen los principios y objetivos de la ayuda pero también sus motivos. De forma muy resumida, podría decirse que los donantes invierten en cooperación al desarrollo por distintos motivos, o por varios a la vez. Los motivos pueden ser altruistas, como la caridad o la solidaridad. Puede desembolsarse ayuda en aras del bien común o para la construcción de bienes públicos globales (o para la contención de males públicos globales). Por último, los motivos de la ayuda pueden ser egoístas o de interés nacional (incluyendo, entre otros, el retorno económico).

El mismo análisis de contenidos revela que el número de referencias a los motivos de la ayuda es muy reducido si se compara con el número de referencias a los objetivos de la ayuda. Sólo hay seis referencias a estos motivos en el Consenso de 2006 y cinco en el más reciente Consenso de 2017. Las referencias a los dos

\footnotetext{
${ }^{5}$ Los detalles metodológicos de la definición de los paradigmas, el diseño de códigos y su aplicación a ambos textos pueden encontrarse en Olivié y Pérez (2021).
} 
conjuntos de motivos sobre la solidaridad, la responsabilidad y/o la obligación moral, por un lado, y los bienes comunes, los esfuerzos comunes, los intereses comunes y/o los bienes públicos mundiales, por otro, están equilibradas (tres y dos en el primer y segundo documento, respectivamente). Sólo se hace una referencia al interés propio, en el primer documento.

Estos resultados no coinciden enteramente con las conclusiones de estudios anteriores sobre la seguridad en el discurso de la ayuda en Europa que observan un importante y reciente giro securitario en la ayuda europea (Keukeleire y Raube, 2013; Furness y Gänzle, 2016, Brown, Grävingholt, y Raddatz, 2016). Dichos resultados de codificación, en cambio, denotan una cierta estabilidad en el discurso de la ayuda europea, antes y después de las policrisis, en torno a los paradigmas de desarrollo social y sostenible, aunque con elementos geoestratégicos y geopolíticos que podríamos asociar a una cierta securitización de la ayuda (en cualquier caso, previa a 2006). Esta misma estabilidad se refleja en los motivos de la ayuda, que se argumenta desde el altruismo y la construcción del bien común. La explicación a esta estabilidad podría estar en el hecho de que los consensos sobre el desarrollo fueron el resultado de negociaciones entre los $28 \mathrm{EM}$, así como las instituciones de la UE, lo que refleja sensibilidades políticas muy variadas con respecto a la acción exterior en su conjunto, y a la ayuda en particular, quedando un resultado final de consenso relativamente estable.

\subsection{El cómo: agenda de eficacia de la ayuda y coherencia de políticas para el desarrollo}

Tanto los consensos de desarrollo como otros documentos estratégicos de la cooperación europea prestan particular atención a la forma en cómo debe canalizarse la ayuda.

En este sentido, tuvo un papel particular en moldear la ayuda europea (y su discurso) la denominada Agenda de Eficacia de la Ayuda (AEA). Los contenidos de la actual AEA son el resultado de una evolución desde el foro de alto nivel de Roma promovido por la OCDE en 2003. En aquella primera reunión, se esbozaron los principios fundamentales de la ayuda: alineación con los procedimientos y objetivos de los países socios, y enfoque en el impacto y los resultados de la cooperación al desarrollo. Con matices y reinterpretaciones, éstos se han mantenido como la columna vertebral de la AEA hasta ahora, que se listan en los cuatro principios siguientes: (1) la orientación a resultados, (2) la apropiación de las prioridades de desarrollo por parte de los países socios, (3) los partenariados de desarrollo 
inclusivos y (4) la transparencia y rendición de cuentas mutua. Tras la reunión de Busan de 2011 , la AEA se institucionalizó en la Alianza Global para la Cooperación Eficaz al Desarrollo (GPEDC, por sus siglas en inglés), una plataforma multi-actor que integra tanto a donantes como a socios (en particular, Brasil, China e India) y a organizaciones multilaterales (Abdel-Malek 2015).

Podría decirse que, aunque la AEA ha ido perdiendo fuelle, sigue encapsulando el pensamiento ortodoxo predominante en relación con las normas de ayuda (con un visible sesgo occidental y europeo) (Brown 2017; Keijzer y Black 2020). Esto se muestra, por ejemplo, en la importancia de la apropiación, las asociaciones o la transparencia en la actual Agenda 2030 y los Objetivos de Desarrollo Sostenible (ODS).

El peso asignado a la AEA por parte de la UE llegó a ser tal que esta agenda copaba prácticamente el texto del Tratado de Lisboa dedicado a la ayuda al desarrollo (Unión Europea, 2007). En línea con esto, los principios de eficacia se subrayan en ambos consensos de desarrollo y se utiliza como principal argumento para distintas iniciativas que se podrían calificar de comunitarizadoras de facto. Así las cosas, la eficacia se ha esgrimido como el principal argumento para las iniciativas de coordinación o, incluso integración, impulsadas desde Bruselas.

También resultaría esencial para incrementar la eficacia de la ayuda la coherencia de políticas para el desarrollo (CPD) o, en su versión más reciente, la coherencia de políticas para el desarrollo sostenible (CPDS). La CPD forma parte, también, del tratado constitutivo (ibid.), después de haberle dedicado una comunicación en 2005 (Comisión Europea, 2005). En dicha comunicación se identifican una serie de políticas clave a alinear, y esto en las áreas de comercio, medio ambiente, seguridad, agricultura, pesca, dimensión social de la globalización y la promoción de empleo y del trabajo digno, migraciones, investigación e innovación, sociedad de la información, transporte, y energía. 


\section{La ayuda europea: canales, instrumentos y patrón geográfico}

\section{I. Los canales y los instrumentos}

\section{I.I. Vecindad, Desarrollo y Cooperación Internacional}

Un breve repaso de la historia reciente de los instrumentos de la Unión Europea para la consecución de estos objetivos permite ver cómo, tal y como se destacaba anteriormente, el paradigma del desarrollo social protagonizado por los ODM ha dado paso no solo a uno de desarrollo sostenible sustentado por los ODS, sino también a la gradual intensificación de un enfoque geopolítico.

El Fondo de Desarrollo (FED) fue establecido por primera vez en 1959, y se han sucedido desde entonces 11 ciclos del mismo, de aproximadamente cinco años cada uno, siendo el undécimo constituido por el Acuerdo de Cotonú revisado para el período 2014-2020. Este último FED estaba dotado de 30.506 millones de euros para el período 2014-2020 (Unión Europea, Consejo, 2015). Esta herramienta brindaba ayuda financiera y técnica a la cooperación al desarrollo en los Estados de África, el Caribe y el Pacífico (ACP) y en los Países y Territorios de Ultramar (PTU). Sin embargo, se ha mantenido siempre fuera de los presupuestos generales de la UE. En su lugar, eran los EM los que lo financiaban (con contribuciones adicionales del $\mathrm{BEI}$ ), por lo que contaba con normas financieras y comité propios.

El Instrumento Europeo de Vecindad y Asociación (IEVA), establecido en 2007 y sucedido por el Instrumento Europeo de Vecindad (IEV) en 2014, se constituyó como el instrumento de financiación de la Política Europea de Vecindad (PEV) (Europa, Parlamento y Europa, Consejo, 2014b). Dicha políitica creaba un espacio de cooperación con los países socios de la vecindad para promover su desarrollo político, económico y social, así como la mayor integración regional.

Elementos como la buena gobernanza, el Estado de derecho, el fomento de la democracia y el desarrollo sostenible son comunes a ambas versiones, pero la lucha contra la pobreza en estos países pasó a un segundo plano en 2014. Además, es destacable que en 2007 se incluía a Rusia como país socio en el marco de cooperación de este Instrumento, mientras que en 2014 no se encontraba ya entre los países socios de la PEV, aunque se admitía la posibilidad de su participación. El presupuesto de este Instrumento para el período 2014-2020 era de 15.433 millones de euros (Comisión Europea, s.f.). 
El Instrumento de Financiación de la Cooperación al Desarrollo unificaba a su vez, en 2007, varios instrumentos geográficos y temáticos con el objetivo de reducir la pobreza, impulsar el desarrollo económico y social sostenible y respaldar la inserción de los países en desarrollo en la economía mundial; todo ello en el marco de los Objetivos de Desarrollo del Milenio (Europa, Parlamento y Europa, Consejo, 2006a). Si el foco geográfico del FED eran los países de África, el Caribe y el Pacífico y los Países y Territorios de Ultramar, y el del IEVA/IEV eran los países de la vecindad, el ICD brindaba ayuda especialmente en Asia, Asia Central, Oriente Medio, América Latina y Sudáfrica, con un presupuesto de 19.600 millones de euros para el período 2014-2020 (Europa, Parlamento y Europa, Consejo, 2014c).

Al renovar el ICD en 2014, el Parlamento y el Consejo insistían en la reducción de la pobreza y el desarrollo económico y social sostenible, así como en "la democracia, el Estado de derecho, la buena gobernanza, los derechos humanos y los principios pertinentes del Derecho Internacional" (ibid., p. 48), ya presentes en 2007, aunque con menos protagonismo. Si bien la cooperación en materia de migración y asilo se incluía entre los programas temáticos del Instrumento, el mayor énfasis en los otros objetivos era notable en ambos períodos del mismo. También se aprecian las alusiones a la buena gobernanza y los derechos humanos que ya aparecían en el Consenso de 2006, aunque no aún en los ODM.

Constituido en 2014, el Instrumento de la UE en pro de la Estabilidad y la Paz sustituía al previo Instrumento de Estabilidad para proporcionar ayuda técnica y financiera en respuesta a crisis, conflictos y amenazas globales, con un presupuesto de 2.339 millones de euros para el período 2014-2020 (Comisión Europea, s.f.). La creación de este instrumento reconocía el nexo entre desarrollo y seguridad, por lo que preveía la complementariedad entre los recursos de cooperación, ayuda humanitaria y seguridad. En línea con el incremento de las prioridades de seguridad mencionadas anteriormente, se introducía en 2014 una modificación al reglamento que permitía destinar ayuda al desarrollo de las capacidades militares locales, siempre y cuando fuera con el único objetivo de garantizar la seguridad para poder implementar las actividades de desarrollo (Europa, Parlamento y Europa, Consejo, 2014a).

Con el nuevo marco financiero plurianual de la UE para el período 2021-2027, todos estos instrumentos $-y$ algunos más- han expirado y han sido fusionados en uno solo, el Instrumento de Vecindad, Desarrollo y Cooperación Internacional ( $\mathrm{NDICl}$, por sus siglas en inglés). El objetivo del NDICl se establece como "defender y promover los intereses y valores de la Unión en el mundo para perseguir los objetivos y principios de su acción exterior" (Comisión Europea, 2018, p. 1), dado el 
incremento de la inseguridad, los conflictos, la desigualdad y la presión migratoria y la persistencia de la pobreza y la fragilidad en el contexto actual. Las nociones de seguridad y de intereses propios están, por tanto, incluidas explícitamente en la conformación de este Instrumento con el mismo o más énfasis que el objetivo tradicional por excelencia de la erradicación de la pobreza.

El nuevo Instrumento abarca, por lo tanto, la diversidad de focos geográficos y temáticos que antes competían a instrumentos separados. Así, con un presupuesto total de 79.500 millones de euros para el período 2021-2027, el NDICl tiene un presupuesto de 60.380 millones de euros para programas geográficos, de los cuales 19.320 millones están asignados a la Vecindad, 29.180 millones al África Sub-Sahariana, 8.480 millones a Asia y el Pacífico, y 3.390 millones a las Américas y el Caribe (Comisión Europea, 2021). Reúne además los objetivos en materias de derechos humanos y democracia, organizaciones de la sociedad civil, paz, estabilidad y prevención de conflictos y retos globales, con 6.360 millones de euros de presupuesto para estos programas temáticos (ibid.). Se reserva, asimismo, 3.180 millones de euros para acciones de respuesta rápida (ibid.).

La creación de este instrumento para sustituir a los anteriores refleja el giro geopolítico de la UE como actor global, ya que a partir de 2021 no existe ya un Instrumento dedicado a la cooperación al desarrollo que no esté también orientado a la seguridad, particularmente en la vecindad europea. De hecho, el reglamento del NDICl incluye una sección específica para la regulación y ejecución de las competencias de vecindad, algo que no hace para ninguna otra área.

Por otra parte, la fusión de tantos instrumentos en uno solo -y especialmente la incorporación del FED, hasta ahora extrapresupuestario-, se argumenta por parte de la UE por motivos de eficacia y coherencia. En este sentido cabe destacar una tendencia hacia la homogeneización de la gobernanza de distintos instrumentos de la ayuda europea: si los comités de cada instrumento tenían, previamente a 2014, su propio reglamento, además de un escueto reglamento común, éstos pasan después a estar regulados por un único reglamento aplicable a todos ellos. Además, la creación del NDICl podría estar mostrando la voluntad de la Comisión por aumentar el control sobre unos fondos de ayuda canalizados enteramente por vía presupuestaria, en la que el gobierno de los EM se diluye.

En términos más amplios, podría interpretarse en la lógica de una creciente comunitarización de facto de la ayuda europea, que se pone también de manifiesto en otras iniciativas como Team Europe, la programación conjunta o la cooperación delegada. Por último, con una dotación presupuestaria para el conjunto del NDICI 
que supera la suma de los presupuestos, en el anterior ciclo fiscal, de todas las herramientas que pasa a integrar (y esto a pesar de la salida del Reino Unido de la UE y de la integración en presupuestos del FED), el tránsito a esta herramienta también revela la voluntad de la UE de consolidarse como un actor global clave en el ámbito del desarrollo.

\section{I.2. El Fondo Europeo de Desarrollo Sostenible (Plus)}

El Fondo Europeo de Desarrollo Sostenible (FEDS) se estableció en 2017 en paralelo a otros instrumentos (incluido el FED) para gestionar y brindar apoyo financiero a inversiones en pos de los ODS en África y los países vecinos de la UE, especialmente el de la erradicación de la pobreza (Europa, Parlamento y Europa, Consejo, 2017). De esta manera, además, se abordarían también las causas de la migración. Así, el FEDS canalizaba las propuestas de inversión de distintos actores -entidades financieras o inversores públicos o privados- a través de dos plataformas regionales de inversión que colaboraban con la Comisión Europea para establecer las prioridades en este ámbito.

El FEDS contaba, además, con la Garantía del FEDS, que proporcionaba financiación adicional y riesgo compartido a posibles inversores. La Unión debía dotarla de 1.500 millones de euros, importe que no podría por tanto exceder en el total de los pagos que realizara. También contaba con el Fondo de Garantía del FEDS, una reserva de liquidez a la que la Unión dotaría de 750 millones de euros para cubrir posibles pérdidas, según lo estipulado en el acuerdo de garantía correspondiente (ibid.).

El marco financiero plurianual para 2021-2027 ha sustituido al FEDS por el FEDS+ para proveer ayuda financiera y garantías presupuestarias en todo el mundo (ya no solo en África y la vecindad de la UE), en apoyo del Plan de Inversiones Exteriores. Se trata de una "ventanilla única" (Comisión Europea, 2018, p. 25) para apoyar inversiones que ayuden a la consecución de los ODS, con un enfoque especial en el impulso de la resiliencia en países socios, la creación de puestos de trabajo dignos, el apoyo a las PYMEs y el aborde de las "causas profundas socioeconómicas específicas de la migración irregular" (ibid., p. 24), además de la lucha contra la pobreza y el desarrollo económico y social sostenible. Se ve aquí reflejada una mirada aún más explícita y específica en la migración irregular y las preocupaciones de seguridad en la vecindad.

Con el FEDS+, cuya financiación proviene de la dotación financiera del $\mathrm{NDICl}$, la previa Garantía del FEDS y su Fondo de Garantía del FEDS han sido integradas 
en una nueva Garantía de Acción Exterior que acompaña al FEDS + y que además absorbe el anterior mandato de préstamo en el exterior del Banco Europeo de Inversiones (BEI). De nuevo se puede percibir un intento de simplificación en pos de la eficacia y/o de la integración al concentrar las competencias en un número de organismos más reducido. La siguiente figura muestra la evolución de los principales instrumentos de la UE para la cooperación desde el período 2007-2013 hasta ahora.

\section{I.3. El Banco Europeo de Inversiones}

Como concesor europeo de préstamos y financiación para proyectos alineados con los objetivos de la UE (Unión Europea, s.f.), el BEI ha formado tradicionalmente parte de la constelación de la ayuda al desarrollo europea. Como tal, y a través de su mandato de préstamo en el exterior, tenía un papel importante en la ejecución del FED. Al absorber estas competencias el nuevo FEDS+, que a diferencia del FED sí está incluido en el presupuesto general de la UE, su presencia en la financiación de la cooperación al desarrollo ha disminuido, aunque no desaparecido.

\subsection{Evolución y distribución de la ayuda oficial al desarrollo}

Aquí de nuevo, el hecho de que la UE como donante se pueda definir de dos maneras (las instituciones europeas, únicamente, o el conjunto de éstas y los EM) influye en la calibración del volumen de ayuda europea. Además, la salida del Reino Unido de la Unión en enero de 2020 también influye en el tamaño de la UE como donante.

Las instituciones europeas canalizaron en 2020, según datos aún provisionales de la OCDE, y mediante los canales e instrumentos descritos en este segundo epígrafe, algo más de 19.000 millones de dólares EEUU a precios y tipo de cambio de 2019. La ayuda de las instituciones UE ha crecido, lógicamente, con la propia Unión, partiendo de 1.440 millones en 1970 y acelerándose, como en otros grandes donantes, su crecimiento a partir del año 2000, momento en que los fondos canalizados desde Bruselas se situaron en algo más de 8.000 millones (Gráfico 1). Se trata pues de un donante relevante, de hecho, el tercero por volumen de ayuda del conjunto de los denominados donantes tradicionales (esto es, los que forman parte del Comité de Ayuda al Desarrollo de la OCDE). 


\section{GRÁFICO I. Evolución de los instrumentos de la UE para la cooperación}

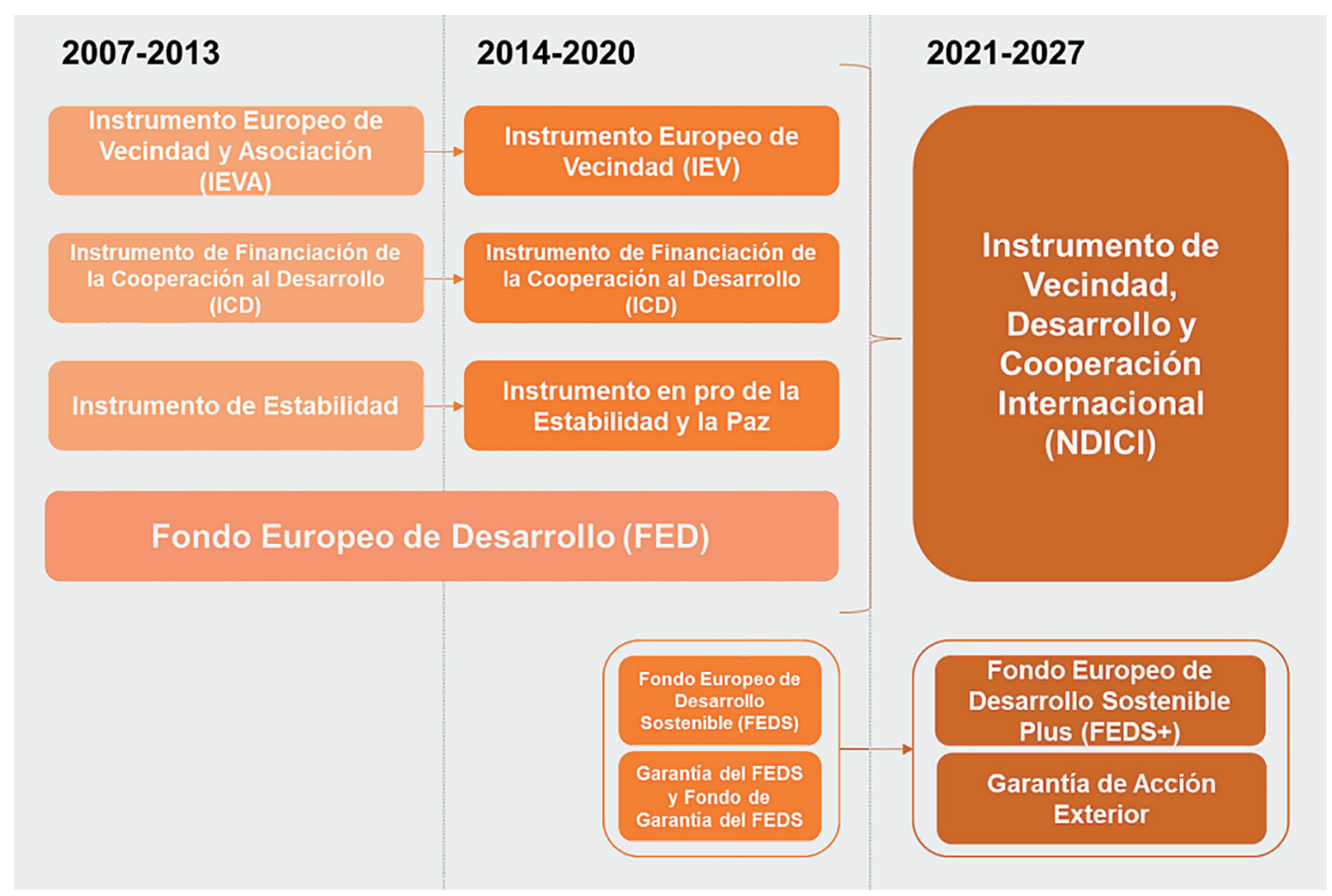

Fuente: Las autoras.

Si a esto sumamos que varios de los EM están también entre los principales donantes mundiales (Alemania, Francia, Países Bajos, hasta recientemente, Reino Unido), nos encontramos con que la UE es el líder mundial indiscutible en el ámbito de la ayuda al desarrollo, al menos en lo que respecta al volumen de ayuda, muy por delante de los otros dos grandes actores globales, que son Estados Unidos y China. Concretamente, según los mismos datos provisionales de la OCDE para 2020 (por lo tanto, ya sin el Reino Unido), la UE aportó el $45 \%$ de la ayuda mundial. Sirva de referencia que el G-7 contribuyó con 76\%, lo que también es reflejo de la concentración de las contribuciones de la ayuda en una lista corta de países.

En lo que se refiere al perfil de las instituciones europeas como donante, la distribución geográfica de la ayuda refleja quizás mejor el enfoque defensivo, reactivo y regional de la estrategia global de 2016 (Unión Europea, 2016) que la narrativa más universalista del consenso de desarrollo de 2017 (Comisión Europea, 2017). Así, la ayuda se dirige fundamentalmente a la vecindad inmediata y a la ampliada: 
$39 \%$ de la ayuda de las instituciones europeas se invierte en Europa, el Norte de África y Oriente medio (Gráfico 2).

\section{GRÁFICO 2. Donantes tradicionales por volumen de ayuda desembolsada en 2020 (miles de millones de dólares EEUU)}

ODA grant equivalent - USD billion (2020)

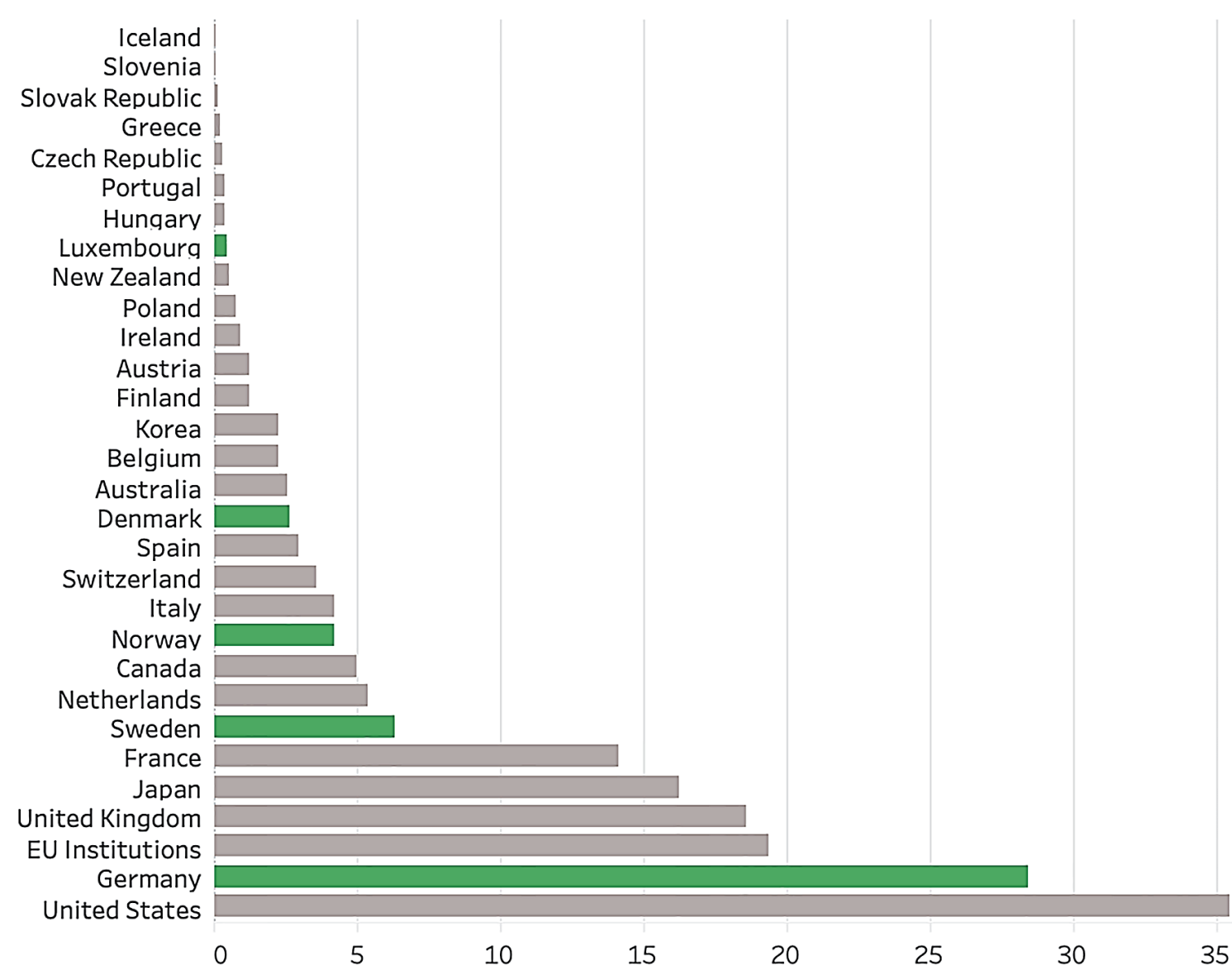

ODA on a grant equivalent measure by members of the OECD Development Assistance Committee (DAC). Preliminary data for 2020.

Fuente: OCDE.

El perfil de la UE en su conjunto (sumando los EM) es tan diverso como lo son los países e instituciones que lo definen. Por este motivo, no sobresale una identidad o perfil claro, con una concentración geográfica o sectorial particular. Los datos de ayuda de la OCDE consolidados por la propia UE revelan, sin embargo, la atención a la vecindad europea y el carácter, en ocasiones, reactivo de la ayuda europea. 


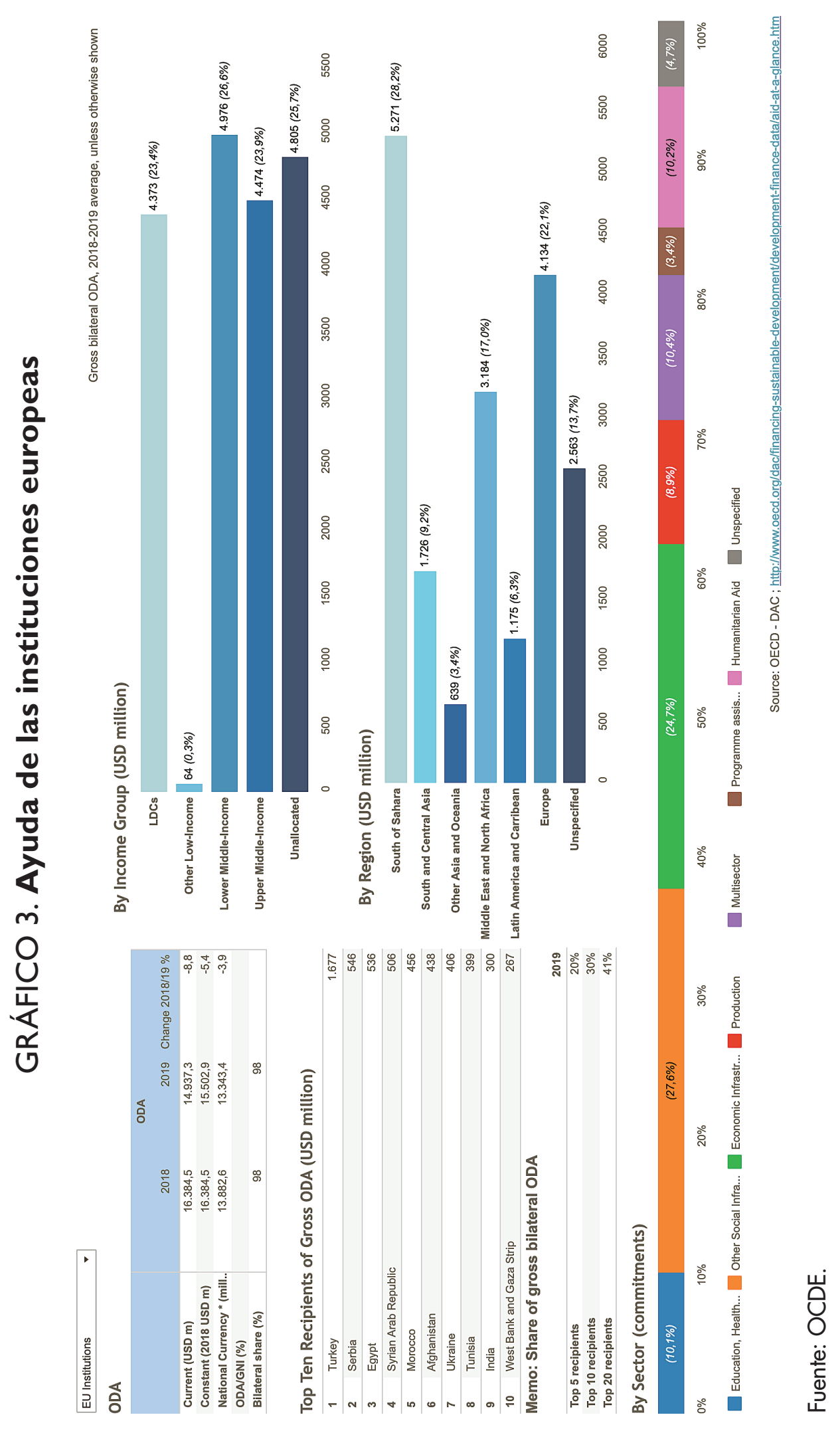


Así, por ejemplo, con cerca de 40.000 millones de euros en ayuda en 2019 (en desembolsos brutos de AOD), Turquía es, muy por delante del segundo país (India), el primer receptor de ayuda europea en 2019. Regionalmente, la prioridad de la vecindad también se hace evidente en el peso de Europa como región de destino de la $A O D$, y esto a pesar del muy dispar nivel de desarrollo respecto de otras regiones receptoras de ayuda como Asia del Sur (Gráfico 3). En esta misma línea, las partidas de apoyo a refugiados en territorio nacional constituyen el primer destino por volumen de desembolso (Gráfico 4).

GRÁFICO 4. Distribución regional de la ayuda (desembolsos de AOD bruta en miles de millones de euros, 2019)

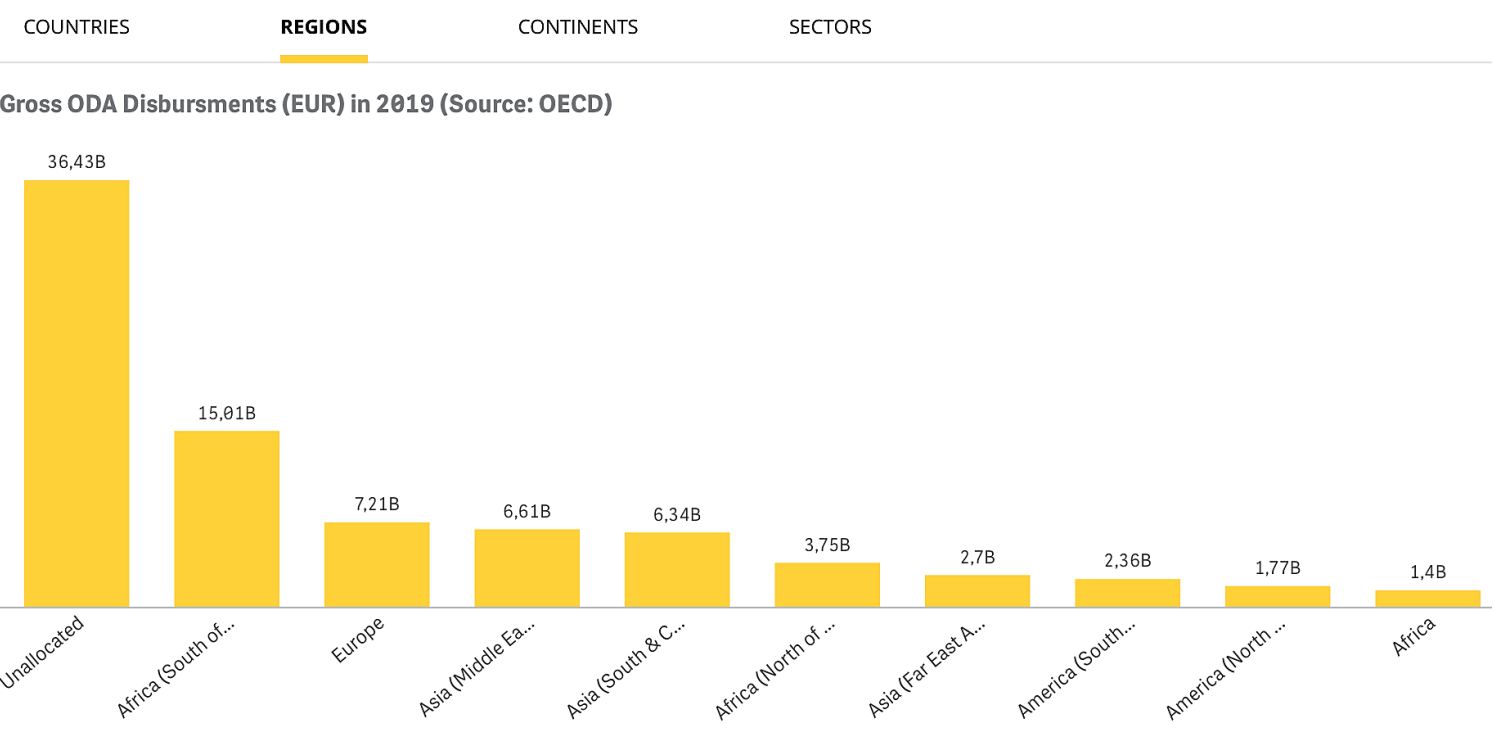

Fuente: UE.

Desde el punto de vista geográfico, la baja prioridad de América Latina se explica también con los muy distintos pesos de los EM dentro de la UE, donde España no destaca como un donante de peso mientras que sí lo hacen otros países, con lazos ya históricos con Asia, como Países Bajos o, hasta principios de 2020, el Reino Unido. 


\section{Gráfico 5. Distribución sectorial de la ayuda (desembolsos de AOD} bruta en miles de millones de euros, 2019)

$\begin{array}{llll}\text { COUNTRIES REGIONS CONTINENTS SECTORS } & \end{array}$

Gross ODA Disbursments (EUR) in 2019 (Source: OECD)

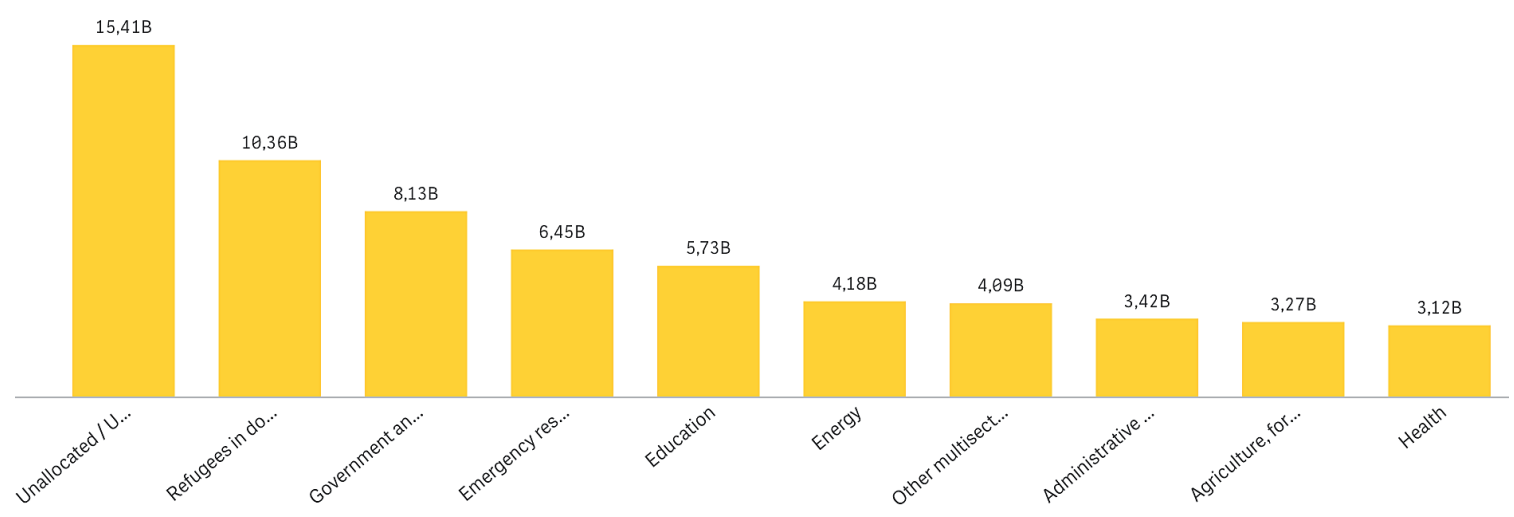

Fuente: UE.

\section{Conclusiones}

Así pues, el perfil de la UE como mayor donante mundial de ayuda al desarrollo ha ido evolucionando, por un lado, de forma paralela a las agendas globales del momento (los ODM y posteriormente los ODS), y por otro, hacia la compaginación de los objetivos tradicionales de solidaridad con objetivos geopolíticos. Estos últimos han generado un mayor énfasis en la promoción de la democracia y la buena gobernanza en los países destinatarios, en el aborde de las causas de la migración, y en un enfoque particular en la vecindad europea; todos ellos reflejados en las asignaciones geográficas y sectoriales de la ayuda. Asimismo, el conjunto de los documentos estratégicos de la UE -fundamentalmente los consensos de desarrollo-, la gradual unificación e integración de los instrumentos de la ayuda y otras iniciativas como Team Europe reflejan una mayor apuesta por la eficacia y la coherencia de la cooperación internacional europea o por la integración como fin último.

Todo ello es paralelo a las tendencias de la acción exterior europea en general, especialmente desde la adopción del Pacto Verde Europeo. Desde la UEy su Servicio Europeo de Acción Exterior (SEAE) se ha llamado a los EM y a las instituciones europeas a alinear toda acción exterior con los objetivos y prioridades comunes del Acuerdo de París sobre cambio climático, que coinciden con los que recoge 
la ayuda al desarrollo europea, a través de la diplomacia climática y energética (Consejo de la UE, 2021). De esta manera, se reconoce la importancia estratégica de exportar los principios climáticos al resto del mundo por motivos humanitarios y de desarrollo, pero también geopolíticos, como la seguridad y la defensa, en reconocimiento de los nexos entre clima, seguridad y desarrollo (ibid.). Así, el $\mathrm{NDICl}$ se contempla también como herramienta vital para esta diplomacia climática y energética, a la que se le atribuye por tanto la responsabilidad de respetar el Acuerdo de París y la coherencia de políticas en toda su ayuda al desarrollo.

Estos intentos de la UE por afianzarse como líder global en materias de desarrollo y medioambiente coinciden con un contexto mundial de pandemia en el que ambos ámbitos cobran una relevancia particular y requieren atención urgente. Se trata, por tanto, de una oportunidad para reforzar su posición, presencia, valores e intereses en todo el mundo. España tiene también la ocasión de aprovechar esta oportunidad, gracias a la actual reforma de su cooperación y al plan de recuperación financiado por los fondos de EU Next Generation, para sumarse a este liderazgo mundial.

\section{Bibliografía}

Abdel-Malek, T. (2015). The Global Partnership for Effective Development Cooperation: Origins, Actions and Future Prospects, Bonn: German Development Institute.

ARTS, K. y DiCKINSON, A. K. (2004). "EU development cooperation: from model to symbol?", en K. Arts y A. K. Dickinson (Eds.) EU development cooperation: from model to symbol, pp. 1-16, Manchester y Nueva York: Manchester University Press.

Brown, S. (2017). "Foreign Aid and National Ownership in Mali and Ghana", Forum for Development Studies 44(3), 335-356.

BroWN, S., GRÄVINGHOLt, J. y RADDATZ, R. (2016). "The Securitization of Foreign Aid: Trends, Explanations and Prospects", en Brown, S. y GrÄVINGHOLT, J. (Eds.) The Securitization of Foreign Aid, 237-255, Londres: Palgrave Macmillan.

COMISIÓN EUROPEA (19 de marzo de 2021). European Commission welcomes the endorsement of the new $€ 79.5$ billion NDICl-Global Europe instrument to support EU's external action [Nota de prensa]. https://ec.europa.eu/commission/ presscorner/detail/en/ip_21_1267 
- (2005). Comunicación de la Comisión al Consejo, al Parlamento Europeo y al Comité Económico y Social Europeo - Coherencia de las Políticas en favor del Desarrollo - Acelerar el avance para cumplir los Objetivos de Desarrollo del Milenio, 12-4-2005, COM(2005) 134 final. https://eur-lex.europa.eu/legal-content/ES/ TXT/?uri=celex\%3A52005DC0134\#document 1

- (2018). Propuesta de Reglamento del Parlamento Europeo y del Consejo por el que se establece el Instrumento de Vecindad, Desarrollo y Cooperación Internacional, 14-6-2018, $\operatorname{COM(2018)~} 460$ final. https://eur-lex.europa.eu/legal-content/ $\mathrm{ES} / \mathrm{TXT} / \mathrm{HTML}$ ? uri=CELEX:52018PC0460\&from=EN

- (s.f.) Heading 4: Global Europe [Página Web]. https://ec.europa.eu/info/ strategy/eu-budget/long-term-eu-budget/2014-2020/funding-programmes/ heading-4-global-europe_es

Consejo de la UE. (25 de enero de 2021). El Consejo adopta unas Conclusiones sobre diplomacia climática y energética (Cumplir la dimensión exterior del Pacto Verde Europeo) [Nota de prensa]. https:// www.consilium.europa.eu/es/press/press-releases/2021/01/25/ council-adopts-conclusions-on-climate-and-energy-diplomacy/

Furness, M. y GänZLE, S. (2016). "The European Union's Development Policy: A Balancing Act between 'a More Comprehensive Approach' and 'Creeping Securitisation'", en Brown, S. y GrÄVINGHOLT, J. (Eds.) The Securitization of Foreign Aid, 138-162, Londres: Palgrave Macmillan.

GRIMM, S. (2006). EU development cooperation: Rebuilding a tanker at sea. Friedrich Ebert Stiffung. https://library.fes.de/pdf-files/iez/global/50421.pdf

KeIJZER, N. y BlACK, D. (2020). "Special issue introduction. Ownership in a post-aid effectiveness era: comparative perspectives", Development Policy Review 38(1), 1-12.

Keukeleire, S. y Raube, K. (2013). "The Security-Development Nexus and Securitization in the EU's Policies towards Developing Countries", Cambridge Review of International Affairs, 26, 1-17.

LE BLANC, D. (2015). "Towards Integration at Last? The Sustainable Development Goals as a Network of Targets", Sustainable Development, 23, 176-187.

LUNDSGAARDE, E. (2011). The challenge from within: New EU donors and European Development Cooperation. (ECD2020 Policy Brief 10). European Develo- 
pment Cooperation to 2020. http://www.edc2020.eu/fileadmin/publications/ EDC2020_-_Policy_Brief_No_10_-_The_Challenge_From_Within__New_Donors_and_European_Development_Cooperation.pdf

OECD (2020). European Union institutions. En Development Co-operation Profiles, Paris: OECD Publishing.

Olvié, I. y Pérez, A. (en prensa). "Whose and what aid securitization? An Analysis of EU aid narratives and flows", Third World Quarterly.

SACHS, J. (2012). "From Millennium Development Goals to Sustainable Development Goals", The Lancet, 379, 2206-2211.

Unión Europea, Consejo, Europa, Parlamento y Europa, Comisión (2006). Declaración conjunta del Consejo y de los Representantes de los Gobiernos de los Estados miembros reunidos en el seno del Consejo, del Parlamento Europeo y de la Comisión sobre la política de desarrollo de la Unión Europea titulada «El consenso europeo sobre desarrollo». Diario Oficial de la Unión Europea, 24-2-2006, C 46, 1-19. https://eur-lex.europa.eu/LexUriServ/LexUriServ.do?uri=OJ:C:2006: 046:0001:0019:ES:PDF

- (2017). Declaración conjunta del Consejo y los representantes de los Gobiernos de los Estados miembros reunidos en el seno del Consejo, del Parlamento Europeo y de la Comisión: El nuevo consenso europeo en materia de desarrollo. Diario Oficial de la Unión Europea, 30-6-2017, C 210, 1-24. https://eur-lex.europa. eu/legal-content/ES/TXT/?uri=celex\%3A42017Y0630\%2801\%29

Unión EUROPEA, Consejo (2015). Reglamento (UE) n² 2015/322 del Consejo, de 2 de marzo de 2015, sobre la aplicación del $11^{\circ}$ Fondo Europeo de Desarrollo. Diario Oficial de la Unión Europea, 3-3-2015, L 58, 1-16. https://www.boe.es/ dove/2015/058/L00001-00016.pdf

Unión Europea, Parlamento y Europa, Consejo (2006a). Reglamento (CE) n 1905/2006 del Parlamento Europeo y del Consejo, de 16 de diciembre de 2006, por el que se establece un Instrumento de Financiación de la Cooperación al Desarrollo. Diario Oficial de la Unión Europea, 27-12-2006, L 378, 41-71. https://eur-lex.europa. eu/legal-content/ES/TXT/?uri=uriserv\%3AOJ.L_.2006.378.01.0041.01.SPA\&to c=OJ\%3AL\%3A2006\%3A378\%3ATOC

- (2006b). Reglamento (CE) $n^{\circ} 1638 / 2006$ del Parlamento Europeo y del Consejo, de 24 de octubre de 2006, por el que se establecen las disposiciones generales 
relativas a la creación de un Instrumento Europeo de Vecindad y Asociación. Diario Oficial de la Unión Europea, 9-1 1-2006, L 310, 1-14. https://eur-lex.europa.eu/ legal-content/ES/TXT/?uri=celex\%3A32006R1638

- (2014a). Reglamento (UE) $n^{\circ}$ 230/2014 del Parlamento Europeo y del Consejo, de 11 de marzo de 2014, por el que se establece un instrumento en pro de la estabilidad y la paz. Diario Oficial de la Unión Europea, 15-3-2014, L 77, 1-10. https://eur-lex.europa.eu/legal-content/ES/TXT/?uri=celex\%3A32014R0230

- (2014b). Reglamento (UE) $n^{\circ} 232 / 2014$ del Parlamento Europeo y del Consejo, de 11 de marzo de 2014, por el que se establece un Instrumento Europeo de Vecindad. Diario Oficial de la Unión Europea, 15-3-2014, L 77, 27-43. https:// eur-lex.europa.eu/legal-content/ES/TXT/?uri=celex\%3A32014R0232

- (2014c). Reglamento (UE) ${ }^{\circ}$ 233/201 4 del Parlamento Europeo y del Consejo, de 11 de marzo de 2014, por el que se establece un Instrumento de Financiación de la Cooperación al Desarrollo para el período 2014-2020. Diario Oficial de la Unión Europea, 15-3-2014, L77, 44-76. hittps://eur-lex.europa.eu/legal-content/ ES/TXT/?uri=celex\%3A32014R0233

- (2017). Reglamento (UE) $n^{\circ} 2017 / 1601$ del Parlamento Europeo y del Consejo, de 26 de septiembre de 2017, por el que se establece el Fondo Europeo de Desarrollo Sostenible (FEDS), la Garantía del FEDS y el Fondo de Garantía del FEDS. Diario Oficial de la Unión Europea, 27-9-2017, L 249, 1-16. https://eurlex.europa.eu/legal-content/ES/TXT/?uri=celex\%3A32017R1601

Unión EUROPEA (2007). Tratado de Lisboa, Lisboa, 13-12-2007. Diario Oficial de la Unión Europea, 17-12-2007, C 306, 1-271. http://publications.europa.eu/ resource/cellar/688a7a98-3110-4ffe-a6b3-8972d8445325.0008.01/DOC_19

- (2016). Una visión común, una actuación conjunta: una Europa más fuerte. Estrategia global para la política exterior y de seguridad de la Unión Europea. Luxemburgo: Oficina de Publicaciones de la Unión Europea.

- (s.f.). Banco Europeo de Inversiones (BEI) [Página Web]. https://europa.eu/ european-union/about-eu/institutions-bodies/european-investment-bank_es. 